Eftychios Siniorakis, Spyridon Arvanitakis, Maximilianos Elkouris, Stamatia Kalogirou, Dimitrios Veldekis, Sotiria Limberi

Sotiria Chest Diseases Hospital, Athens, Greece

\title{
Chronic obstructive pulmonary disease and sacubitril: explore the role of neprilysin before its inhibition
}

\section{Dear Editor,}

Based on the findings of the emblematic PARADIGM-HF study [1], the combination of sacubitril with valsartan emerged as a revolutionary treatment in heart failure with reduced ejection fraction. Sacubitril is a newly introduced neprilysin (NEP) inhibitor (NI) whereas valsartan constitutes a classic angiotensin II receptor blocker (AR). The combination of the two resulted in the prototype of ARNI. Liczek M and colleagues in their interesting article [2] envision the use of ARNI in patients with chronic obstructive pulmonary disease (COPD), especially if they present with pulmonary hypertension. The argument is based on the potential immune-supressory, vasodilatory and bronchodilatory properties of brain natriuretic peptide which is deliberated, after its degradation by NEP is inhibited. We find the proposition of expanding the use of ARNI in COPD challenging, however we would propose a global view of NEP and its substrates before attempting their modulation in COPD.

NEP is an endopeptidase catalyzing among others the degradation of beta amyloid $\mathrm{A} \beta$ (especially the subtypes $\mathrm{A} \beta_{40}$ and $\mathrm{A} \beta_{42}$ ), atrial natriuretic peptide (ANP), and various kinines. NEP and its substrates are implicated in lung function in a manner that assumes particular significance regarding patients with COPD. NEP protects lung vasculature from remodeling, especially under conditions of hypoxia-induced pulmonary hypertension. NEP activity, as well as NEP expression are reduced in alveolar walls and distal vessels in COPD patients [3]. Endothelin-1 and bombesin-like peptides are NEP substrates with potent proliferative and vasoconstrictive properties, while fibroblast growth factor-2 demonstrates angiogenic effects. Pro-inflammatory substrates, such as substance $\mathrm{P}$, contribute to pulmonary vascular remodeling, showing the importance of their degradation by NEP. Depression of NEP in pulmonary artery smooth muscle cells results in increased migration and proliferation, in response to serum and platelet-derived growth factor [4]. Generally speaking, there is an inverse line between NEP and pulmonary vascular remodeling , and this mechanism should be considered when attempting NEP inhibition in COPD.

Bradykinin (BK) is a NEP substrate with known broncho-constrictive properties which impact the course of asthma and COPD. BK is involved in virus-induced inflammatory cell influx and airway hyperactivity [5]. The biological actions of $\mathrm{BK}$ are mediated through $\mathrm{B}_{1}$ and $\mathrm{B}_{2}$ receptors. $B_{1} R$ are overexpressed during respiratory infections, during which an increase of BK activity results in plasma protein extravasation, smooth muscle contraction, release of growth factors and angiogenin from bronchial fibroblasts and finally bronchoconstriction [6]. Inhibiting NEP in patients with asthma and COPD, is expected to induce increased amounts of BK, creating skepticism about their potential bronchial side-effects.

ANP was found to increase after the administration of ARNI. ANP is a form of arrhythmogenic amyloid which accumulates in the heart atria of the elderly, facilitating the development of atrial

Address for correspondence: Spyridon Arvanitakis, Sotiria Chest Diseases Hospital, Athens, Greece, e-mail: sarvanitakis@doctors.net.uk DOI: 10.5603/ARM.2018.0041

Received: 18.09.2018

Copyright (C) 2018 PTChP

ISSN 2451-4934 
fibrillation. In senile human atrial appendage specimens, ANP amyloid fibrils are found around vessels and adjacent to atrial muscle cells [7]. Patients with COPD are vulnerable to atrial arrhythmogenesis especially when hypoxia, hypercapnia, pulmonary hypertension, diastolic dysfunction of left ventricle, oxidative stress and inflammation coexist [8]. ANP deposition in the atria further aggravates these processes, with ATP containing aggregation-prone regions acting as templates for amyloid fibril formation after protein structural rearrangement in the atria. Establishment of atrial fibrillation in COPD patients is an unwelcome risk factor, predisposing to severe cardiorespiratory derangement.

$\mathrm{A} \beta$ is another amyloid, which as a substrate for NEP is increased after ARNI administration. Subtypes $\mathrm{A} \beta_{40}$ and $\mathrm{A} \beta_{42}$ are the main components of amyloid plaques in the brain of patients with Alzheimer's disease (AD). Similarly, increased expression of $A \beta$ is encountered in the retina of patients with progressive loss of their vision, as well as various neoplasms, including lung cancer. $\mathrm{AD}$ is a severe neurodegenerative disease, very common in COPD patients, ranging from 37 to $62 \%$ [9]. In the serum of these patients increased levels of $\mathrm{A} \beta$ are found, even in the absence of apparent cognitive impairment, and they appear to be in a state of dynamic equilibrium with corresponding brain levels [10]. Transport of A $\beta$ from the brain to the lungs and vice versa has been demonstrated, given the disruption of the blood-brain barrier induced to the vascular wall by $A \beta$. In conditions of hypoxia and respiratory infections, $\mathrm{A} \beta$ fibril deposition in COPD lungs further increases, with concomitant elevated levels in the serum and reduced clearance from the brain. An imminent pulmonary dementia is then underway.

ARNI are a new category of hemodynamically acting drugs and their enthusiastic prescription in patients with heart failure may gradually displace other classic therapies. Heart failure, especially under the form of diastolic dysfunction, frequently coexists with COPD. In a comorbid heart-lung context, patients with COPD and pulmonary hy- pertension may become ideal candidates for the prescription of NEP inhibitors, as Liczek et al.[2] suggest. In the meanwhile, the more we enrich our knowledge on NEP and its substrates, the more we will be able to offer efficient, safe and individualized therapies in patients with COPD.

\section{Conflict of interest}

The authors declare no conflict of interest.

\section{References:}

1. Packer M, McMurray JJV, Desai AS, et al. PARADIGM-HF Investigators and Coordinators, PARADIGM-HF Investigators and Committees. Angiotensin-neprilysin inhibition versus enalapril in heart failure. N Engl J Med. 2014; 371(11): 993-1004, doi: 10.1056/NEJMoa1409077, indexed in Pubmed: 25176015.

2. Liczek M, Panek I, Damiański P, et al. Neprilysin inhibitors as a new approach in the treatment of right heart failure in the course of chronic obstructive pulmonary disease. Adv Respir Med. 2018; 86: 183-191, doi: 10.5603/ARM.a2018.0028, indexed in Pubmed: 30110121.

3. Wick MJ, Buesing EJ, Wehling CA, et al. Decreased neprilysin and pulmonary vascular remodeling in chronic obstructive pulmonary disease. Am J Respir Crit Care Med. 2011; 183(3): 330-340, doi: 10.1164/rccm.201002-01540C, indexed in Pubmed: 20813891.

4. Karoor V, Oka M, Walchak SJ, et al. Neprilysin regulates pulmonary artery smooth muscle cell phenotype through a platelet-derived growth factor receptor-dependent mechanism. Hypertension. 2013; 61(4): 921-930, doi: 10.1161/HYPERTENSIONAHA.111.199588, indexed in Pubmed: 23381789.

5. Broadley KJ, Blair AE, Kidd EJ, et al. Bradykinin-induced lung inflammation and bronchoconstriction: role in parainfluenze-3 virus-induced inflammation and airway hyperreactivity. J Pharmacol Exp Ther. 2010; 335(3): 681-692, doi: 10.1124/ jpet.110.171876, indexed in Pubmed: 20847038.

6. Ricciardolo FLM, Sabatini F, Sorbello V, et al. Expression of vascular remodelling markers in relation to bradykinin receptors in asthma and COPD. Thorax. 2013; 68(9): 803-811, doi: 10.1136/thoraxjnl-2012-202741, indexed in Pubmed: 23739138.

7. Millucci L, Paccagnini E, Ghezzi L, et al. Different factors affecting human ANP amyloid aggregation and their implications in congestive heart failure. PLoS One. 2011; 6(7): e21870, doi: 10.1371/journal.pone.0021870, indexed in Pubmed: 21814559.

8. Goudis CA. Chronic obstructive pulmonary disease and atrial fibrillation: An unknown relationship. J Cardiol. 2017; 69(5): 699-705, doi: 10.1016/j.jjcc.2016.12.013, indexed in Pubmed: 28188041.

9. Dodd JW. Lung disease as a determinant of cognitive decline and dementia. Alzheimers Res Ther. 2015; 7(1): 32, doi: 10.1186/s13195-015-0116-3, indexed in Pubmed: 25798202.

10. Bu XL, Cao GQ, Shen LL, et al. Serum Amyloid-Beta Levels are Increased in Patients with Chronic Obstructive Pulmonary Disease. Neurotox Res. 2015; 28(4): 346-351, doi: 10.1007/ s12640-015-9552-x, indexed in Pubmed: 26243505. 